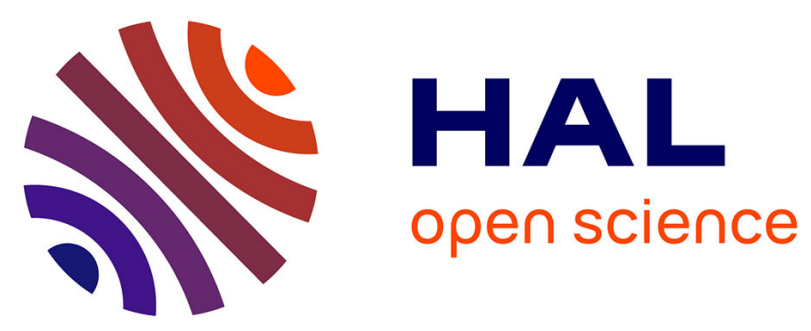

\title{
Optimization of the size of a magnetic microrobot for high throughput handling of micro-objects.
}

Mohamed Dkhil, Aude Bolopion, Stéphane Régnier, Michaël Gauthier

\section{To cite this version:}

Mohamed Dkhil, Aude Bolopion, Stéphane Régnier, Michaël Gauthier. Optimization of the size of a magnetic microrobot for high throughput handling of micro-objects.. IEEE/ASME International Conference on Advanced Intelligent Mechatronics (AIM 2014), Jul 2014, Besançon, France. pp.372377, 10.1109/AIM.2014.6878107 . hal-01313507

\section{HAL Id: hal-01313507 https://hal.science/hal-01313507}

Submitted on 10 May 2016

HAL is a multi-disciplinary open access archive for the deposit and dissemination of scientific research documents, whether they are published or not. The documents may come from teaching and research institutions in France or abroad, or from public or private research centers.
L'archive ouverte pluridisciplinaire HAL, est destinée au dépôt et à la diffusion de documents scientifiques de niveau recherche, publiés ou non, émanant des établissements d'enseignement et de recherche français ou étrangers, des laboratoires publics ou privés. 


\title{
Optimization of the size of a magnetic microrobot for high throughput handling of micro-objects
}

\author{
Mohamed Dkhil ${ }^{(1,2)}$, Aude Bolopion ${ }^{1}$, Stéphane Régnier ${ }^{2}$, Member, IEEE, Michaël Gauthier ${ }^{1}$, Member, IEEE
}

\begin{abstract}
One of the greatest challenges in microrobotic is to handle individually a large number of objects in a short time, for applications such as cell sorting and assembly of microcomponents. This ability to handle a large number of microobjects is directly related to the size of the microrobot. This paper proposes a theoretical study of the size of a magnetic microrobot maximizing its capacity of displacement. It demonstrates that there is an optimal size can be obtained, due to a trade-off between the inertial and the viscous effects. Analytical expressions of the optimal size and the related frequency of motion are derived from a simplified model to highlight the influence of the geometrical and the physical parameters of the magnetic manipulation system such as the viscosity of the liquid and the size of the workspace. A numerical simulation validates the analytical analysis and demonstrates a high displacement capacity of the microrobot (around 100 back and forth motions per second for a robot of around $20 \mu \mathrm{m}$ in water).
\end{abstract}

\section{INTRODUCTION}

Magnetic actuation is used to control robots for a wide range of applications, such as minimally invasive medical procedures [1], cells manipulation [2], [3], [4] and parallel assembly at the microscale [5]. They are based on the use of an artificial magnetic structure called microrobot that can be powered and controlled by a magnetic source [1], [6], [7]. The size of the microrobots ranges from a few micrometers to a few millimeters.

The magnetic microrobots are promising solution to manipulate micro-objects with high precision and rapidity. Indeed, thanks to the development of microfabrication techniques, many complex forms are manufactured (star shape, U shape, rectangular shape, etc.) [6], [7] and investigated on the precise positioning of objects [8] In addition, the ability to handle (position, move, sort, characterize, etc.) a large number of micro-objects is crucial. For example, biologists would greatly benefit from a microrobotic system able to characterize and to sort each cell of a whole population individually according to its mechanical and/or electrical properties. The number of cells in a population can reach several millions. Sorting a whole population thus requires to perform a large number of elementary operations, such as positioning and moving, per second. This is directly related to the ability of the microrobot to perform a large number of back-and-forth

\footnotetext{
${ }^{1}$ Authors are with FEMTO-ST Institute CNRS UFC/ENSMM/UTBM Automatic Control and Micromechatronic Systems Department, 24 rue Alain Savary, 25000 Besançon, France. mohamed.dkhilefemto-st.fr

${ }^{2}$ Authors are with Institut des Systèmes Intelligents et de Robotique (ISIR), Pierre et Marie Curie University, CNRS UMR 7222, 4 Place Jussieu, 75005 Paris, France.
}

motions per second in its workspace.

No matter its geometrical shape, this dynamic capacity of displacement depends on the size of the microrobot. Indeed, the behavior of a small microrobot is ruled by viscous forces, whereas inertial effects limit the velocity of large microrobots. Therefore, there is an optimal size maximizing the dynamic capacity of displacement.

In this paper the size of the microrobot maximizing the dynamic capacity of displacement is determined. The optimization method can be applied for different shapes of microrobot, but in this work the method is illustrated for a spherical geometry of microrobot. An analytical expression of the optimal size and its related capacity of displacement are derived based on a simplified model. It highlights the influence of the geometrical and physical parameters of the non contact magnetic system on the dynamic capacity of displacement. A numerical simulation is performed to solve the dynamic model of motion without these assumptions to validate the analytical results. It shows good agreement with the analytical expressions. This paper demonstrates that a magnetic microrobot of a size of a few tens of micrometers can perform more than 100 back-and-forth motions per second in its workspace. This work will enable to develop magnetic devices with high capacity of displacement that can be used in the future for any application requiring the handling of a large number of micro-objects.

This paper is organized as follows. In Section II the motion of a ferromagnetic microrobot driven by a magnetic force is modeled. In Section III the analytical expression of the optimal size of the robot is derived based on simplifying assumptions. A numerical simulation which does not require such assumptions is performed in Section IV to validate the analytical approach. Conclusions and perspectives of this work are discussed in Section V.

\section{DyNAMIC MODELING}

\section{A. Non contact magnetic actuation system}

Most of the magnetic actuation systems present similar designs [9] based on several coils. The optimization method proposed in this paper is illustrated on a magnetic device based on four coils using direct propulsion (the movement of the microrobot is produced by the magnetic force). The two pairs of coils enable controlled in-plane displacements (see Figure 1). The microrobot is composed of a ferromagnetic material since its magnetization is higher than 
paramagnetic or diamagnetic materials, leading to higher magnetic forces and thus higher velocities [6]. It is placed in a workspace filled with a liquid. An ambient environment would present a smaller viscosity but the adhesion force between the robot and the substrate would be high, leading to a poor reproducibility of the control. In addition most of the current systems are in liquid environments since it enables biomedical applications [8], [7], [10]. The workspace is located in the center of the magnetic actuation device. A non uniform magnetic field is created by applying a current in the coils. The size of the workspace is closely related to the size of the robot. At the microscale practical considerations also limit the size of the workspace since imaging small particles necessitate a high magnification lens which presents a small field of view. In that work it will be considered that the size of the workspace is proportional to the size of the robot.

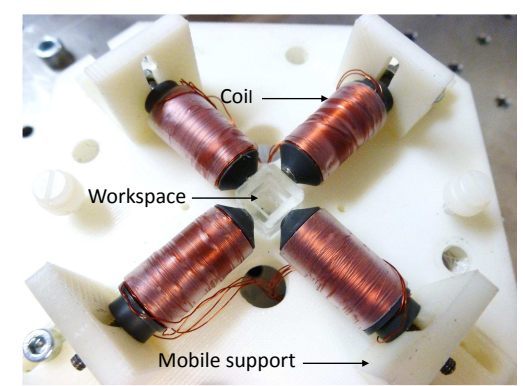

Fig. 1. Non contact magnetic actuation device: four coils create a magnetic force to control the position of the robot. They are placed on a mobile support to adapt the gap between the coils to the size of the workspace.

To determine the size of the microrobot that enables the highest dynamic capacity of displacement a reference trajectory along one axis will be considered (see Figure 2). The microrobot is placed at one extremity of the workspace, on the coil axis $(o, \vec{x})$ (position $A$ ). It is first moved to position $B$, at the other extremity of the workspace, using the coil 1. Then it is moved back to position $A$ using coil 2. It is considered that the same time $T$ is needed to perform each trajectory $A \rightarrow B$ and $B \rightarrow A$. The total trajectory is thus performed in a time $2 T$.

The microrobot used is spherical (radius $r$ ) and composed of ferromagnetic material. The workspace is located at a distance $d$ from the coils. Its size $w$ is proportional to the one of the microrobot: $w=a r$. Two criteria are used to evaluate the performance of the microrobot: (i) the average velocity $V_{m}$ needed to perform the round trip $A \rightarrow B \rightarrow A$, (ii) the related frequency of motion $f=\frac{1}{2 T}$ :

$$
\left\{\begin{aligned}
V_{m} & =\frac{x(T)}{T}=\frac{a r}{T}, \\
f & =\frac{1}{2 T}=\frac{V_{m}}{2 a r} .
\end{aligned}\right.
$$

A dynamic model will be derive to determine these two criteria with respect to the radius $r$.

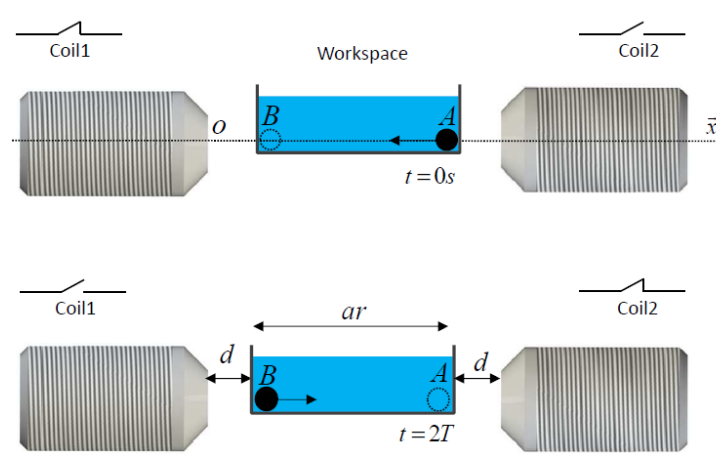

Fig. 2. Side view of the magnetic device: A reference trajectory along one axis is considered to evaluate the performance of the microrobot. The microrobot is placed at one extremity of the workspace (position $A$ ) and it is moved to position $B$, at the other extremity of the workspace, using the coil 1. It is then moved back to $A$ using coil 2. $a$ : scale factor, $d$ : offset distance, $r$ : radius of the microrobot, $T$ : time needed to perform the trajectory $A \rightarrow B$.

\section{B. Dynamic model}

Since the displacement $A \rightarrow B$ and $B \rightarrow A$ is symmetric the analysis will be focused on the motion created by one coil only. Figure 3 shows the configuration of the system when the coil 1 is switched on.

The forces applied to the microrobots are: (i) the magnetic force produced by the coils, (ii) the drag force, (iii) the gravitational force compensated by normal force of the substrate. It is assumed that the adhesion force between the microrobot and the substrate is negligible since it is in liquid environments.

The magnetic force $\vec{F}_{m}$ applied to a ferromagnetic spherical microrobot, is given by [11]:

$$
\vec{F}_{m}=\frac{4}{3} \pi r^{3}(\vec{M} \cdot \nabla) \vec{B},
$$

where $\vec{B}$ is the external magnetic field vector which depends on the position of the microrobot $(x, y, z)$ and the current applied in the coils, $\vec{M}$ is the magnetization vector and $r$ is the radius of the microrobot. Soft magnetic materials (e.g: nickel and iron) are characterized by their low coercivity $\left(H_{c}<\right.$ $\left.10^{3} \mathrm{~A} / \mathrm{m}\right)$. They can be easily magnetized by external fields [11]. It is thus assumed in this work that the magnetic field produced by the coils induces values of external excitation $H$ higher than $H_{c}$, and thus that the ferromagnetic material is saturated. This hypothesis will be discussed in the numerical analysis.

The drag force applied to a spherical microrobot depends on the density of the fluid $\rho_{f}$ and its dynamic viscosity $\eta$. For Reynolds numbers smaller than 1000 it is defined by the Schiller-Naumann model [12]:

$$
\vec{F}_{d}=-k_{1} \vec{V}-k_{2}\|\vec{V}\|^{1.687}\left(\frac{\vec{V}}{\|\vec{V}\|}\right),
$$

where $k_{1}=6 \pi r \eta, k_{2}=1.448 \pi r^{1.687} \eta^{0.313} \rho_{f}^{0.687}$ and ${ }^{t} \vec{V}=(\dot{x}, \dot{y}, \dot{z})$ is the vector representing the velocity of the microrobot.

Using the second Newton's law and a projection along the 


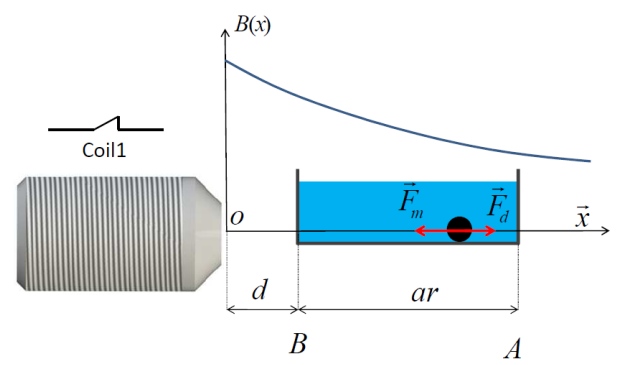

Fig. 3. Configuration of the system when the coil 1 is actuated : the workspace is located at a distance $d$ from coil 1 and the microrobot moves under the action of the magnetic field $B$ to cover the distance $w=a r$ from $A$ to $B . F_{m}$ and $F_{d}$ are the magnetic and the drag force respectively.

$(o, \vec{x})$ axis, the motion the microrobot is given by:

$$
m \ddot{x}=F_{m}(x, i)+F_{d}(\dot{x}),
$$

where $m$ is the mass of the microrobot, $\ddot{x}$ is its acceleration and $i$ is the current applied in the coil. Based on equations (2), (3) and (4) the motion of the microrobot is ruled by:

$$
\ddot{x}+\left(\frac{1}{\tau_{1}}\right) \dot{x}+\left(\frac{1}{\tau_{2}}\right) \dot{x}^{1.687}=\left(\frac{M_{s}}{\rho_{p}}\right) \frac{\partial B(x, i)}{\partial x},
$$

where $\tau_{n}=\frac{m}{k_{n}}, n=(1,2), \rho_{p}$ is the density of the microrobot and $M_{s}$ is the value of the saturated magnetization of the microrobot.

The behavior of the microrobot is given by equation (5). In order to study the impact of the microrobot size on the dynamic behavior, analytical resolution of the equation (5) will be performed under some assumptions in Section III.

\section{ANALyTiCAL ANALYSis OF THE EQUATION OF MOTION}

In order to derive an analytical expression of the average velocity $V_{m}$ and of the motion frequency $f$ defined in equation (1) from the dynamic model (5) some assumptions must be made:

1) The fluid flow is considered as a Stokes flow, thus the drag force is given by :

$$
F_{d}(\dot{x})=-6 \pi r \eta \dot{x}=-k_{1} \dot{x}
$$

2) The magnetic field gradient is considered linear in function of the input current $i$ [13]. Thus the variation of the magnetic field $B$ as a function of the position $x$ of the microrobot can be expressed using the Maxwell equations as follows:

$$
\frac{\partial B(x, i)}{\partial x}=i \cdot g(x)
$$

where $g(x)$ is a function that characterizes the non linearity of the magnetic field gradient $\nabla B$ with respect to the position $x$.

3) The magnetic field gradient is considered to be linear around the operating point $\left(x_{0}=d+a r, i=i_{0}\right)$. Thus, based on a Taylor expansion, the linearized expression of (7) is given by:

$$
\begin{aligned}
\frac{\partial B(x, i)}{\partial x} & \left.\simeq\left(x-x_{0}\right) \frac{\partial(i \cdot g(x))}{\partial x}\right|_{\left(x_{0}, i_{0}\right)}+i \cdot g\left(x_{0}\right) \\
& =\left.\left(x-x_{0}\right) \frac{\partial^{2} B(x, i)}{\partial x^{2}}\right|_{\left(x_{0}, i_{0}\right)}+\left.\left(\frac{i}{i_{0}}\right) \frac{\partial B(x, i)}{\partial x}\right|_{\left(x_{0}, i_{0}\right)} .
\end{aligned}
$$

These assumptions will be discussed in Section IV. The analytical results will be compared to the numerical simulations of the equation of motion performed without making these assumptions. Based on the above equations the simplified dynamic model obtained is expressed as follows:

$$
\Delta \ddot{x}+\left(\frac{1}{\tau_{1}}\right) \Delta \dot{x}-\alpha \Delta x=\beta I
$$

where:

$$
\left\{\begin{aligned}
\alpha & =\left.\left(\frac{M_{s}}{\rho_{p}}\right) \frac{\partial^{2} B(x, i)}{\partial x^{2}}\right|_{\left(x_{0}, i_{0}\right)}>0 \\
\beta & =\left.\left(\frac{M_{s}}{\rho_{p}}\right) \frac{\partial B(x, i)}{\partial x}\right|_{\left(x_{0}, i_{0}\right)}<0 \\
I & =\left(\frac{i}{i_{0}}\right) \text { and } \Delta x=x-x_{0} .
\end{aligned}\right.
$$

The parameters $\alpha$ and $\beta$ define the intensity of the magnetic force which depends on the operating point.

In order to create the magnetic force, the current in the coils is controlled. Several signals of current can be applied to perform the trajectory $A \rightarrow B$. In this work a constant value of current is used to illustrate the optimization method. Using the Laplace transform then its inverse properties, the position $x(t)$ when the input current is $i=i_{0}$ is given by:

$x(t)=x_{0}+\frac{\beta}{\lambda^{+}-\lambda^{-}}\left[\left(\frac{\exp \left(\lambda^{+} t\right)-1}{\lambda^{+}}\right)-\left(\frac{\exp \left(\lambda^{-} t\right)-1}{\lambda^{-}}\right)\right]$,

where $\lambda^{(+,-)}=\frac{-1 \mp \sqrt{1+4 \alpha \tau_{1}^{2}}}{2 \tau_{1}}$ are the poles of system. Equation (12) presents two behaviors for spheres of small or large radius detailed in the following paragraphs. The optimal radius $r_{o p}$ can be approximated by the limit between these two behaviors.

\section{A. First behavior - small radius}

When the radius $r$ of the microrobot tends to 0 , the position $x(t)$ can be simplified as follows:

$$
x(t)=x_{0}+\frac{\beta_{0}}{\alpha_{0}}\left(\exp \left(\alpha_{0} \tau_{1} t\right)-1\right),
$$

where $\alpha_{0}$ and $\beta_{0}$ are respectively the values of $\alpha$ and $\beta$ when $x_{0}=d$. This expression represents the solution of the dynamic model given by:

$$
\frac{1}{\tau_{1}} \Delta \dot{x}-\alpha_{0} \Delta x=\beta_{0} I .
$$

This equation could also be obtained from the general dynamic model (10) by neglecting the inertial term. It can 
be concluded that the inertial force is negligible for small microrobots.

Based on the Taylor expansion $\exp (h) \simeq 1+h$ and the expression of $\tau_{1}$ and $\beta_{0}$, the average velocity and the frequency of the motion can be expressed as follows:

$$
V_{m} \simeq-\beta_{0} \tau_{1}=\left(-\frac{2 \rho_{p} \beta_{0}}{9 \eta}\right) r^{2} \text { and } f \simeq\left(-\frac{\beta_{0} \rho_{p}}{9 \eta a}\right) r .
$$

Note that $\beta_{0}<0$ ( see (11)), $V_{m}$ and $f$ are positive functions. The modification of the workspace size (parameter $a$ ) does not change the average velocity. This is due to the high dynamic behavior: the microrobot reaches a permanent velocity instantaneously because the inertial force is negligible. The frequency of motion $f$ decreases while the workspace size increases, and it increases linearly with respect to the radius of the microrobot.

\section{B. Second behavior-large radius}

Using an asymptotic expansion of the poles $\lambda^{+}$and $\lambda^{-}$ for a high radius, $x(t)$ can be expressed as follows:

$$
x(t)=x_{0}+\frac{\beta}{\alpha}(\cosh (\sqrt{\alpha} t)-1) .
$$

This expression represents the solution of the dynamic model given by:

$$
\Delta \ddot{x}-\alpha \Delta x=\beta I .
$$

This model can also be obtained from the general dynamic model (10) if the viscous force is neglected. It can be concluded that the viscous force is negligible for the spheres with a large radius.

Based on the Taylor expansion $\cosh (h) \simeq 1+\frac{h^{2}}{2}$ in (16) the average velocity and the frequency of motion can be expressed as:

$$
V_{m} \simeq \sqrt{-\frac{\beta a r}{2}} \text { and } f \simeq \sqrt{-\frac{\beta}{8 a r}} .
$$

Note that $\beta<0$ (see (11)), $V_{m}$ and $f$ are positive functions. The average velocity (resp. the frequency of motion) increases (resp. decreases) with respect to the workspace size and the microrobot radius.

\section{Optimal radius}

Two different behaviors can thus be derived from equations (15) and (18): for small radius microrobots the frequency of motion $f$ increases when the radius $r$ increases (equation (15)) while for large ones the frequency decreases when the radius increases (equation (18)). The maximum frequency of motion is obtained for an optimal radius $r_{o p}$ which is approximated by the intersection of the curves of $f$ for the small and large radius. Based on (15) and (18), the values of this optimal radius can be computed by solving the equation:

$$
8\left(\beta_{0} \rho_{p}\right)^{2} r^{3}+81 \eta^{2} a \beta=0 .
$$

To get an analytical solution of this equation (19), the approximation that the magnetic field gradient is constant is made, with $\beta=\beta_{0}$. This approximation will be discussed in the next section. Under these conditions the optimal radius can be expressed by:

$$
r_{o p}=\left(-\frac{81 a \eta^{2}}{8 \beta_{0} \rho_{p}^{2}}\right)^{\frac{1}{3}}
$$

TABLE I

NUMERICAL VALUES USED FOR THE SIMULATIONS

\begin{tabular}{|l|l|}
\hline Microrobot & Medium \\
\hline Material: nickel & - Liquid: water \\
$\rho_{p}=8902\left(\mathrm{~kg} / \mathrm{m}^{3}\right)$ & $\rho_{f}=1000\left(\mathrm{~kg} / \mathrm{m}^{3}\right)$ \\
$M_{s}=4.7710^{5}(\mathrm{~A} / \mathrm{m})$ & $\eta_{f}=10^{-3}$ Pa.s \\
& - Liquid: blood \\
& $\rho_{f}=1066\left(\mathrm{~kg} / \mathrm{m}^{3}\right)$ \\
& $\eta_{f}=6.10^{-3}$ Pa.s \\
\hline
\end{tabular}

Using the parameters presented in Table I and for a workspace size that is ten times the dimension of the microrobot $(a=10)$, placed at a distance $d=4 \mathrm{~mm}$ of the coil and for a magnetic field gradient $\nabla B=3 \mathrm{~T} / \mathrm{m}$ (which represents $\beta_{0}=-160 \mathrm{mATkg}^{-1}$ from (10)), the optimal radius is $20 \mu \mathrm{m}$ in water and $67 \mu \mathrm{m}$ in blood. This radius of a few tens of micrometers offer the possibility to manipulate objects in microscale, for different applications such as cell sorting or conveying of artificial micro-objects.

\section{NUMERICAL STUDY OF MOTION}

The analytical analysis has highlighted two typical behaviors, for small and large microrobots. The influence of the geometrical and physical parameters of the magnetic actuation system has been derived, and the expression of the optimal radius has been determined. However several assumptions and simplifications have been made. This section presents the numerical analysis of the dynamic model defined in (5), taking into account the actual magnetic field gradient and the Schiller Naumann model of the flow, to evaluate the relevance of the analytical results.

\section{A. Experimental identification of the magnetic field}

In order to perform the numerical simulation, the magnetic field gradient must be determined. Most of the magnetic manipulation systems use ferromagnetic core coils for which there is no analytical expression of the magnetic field. An identification of experimental measurements of the magnetic field is performed in this paper. Alternatively finite element modeling could be used.

Figure 4 shows the experimental system used to measure the magnetic field along the axis of the coil. Measurements were made using a teslameter (F.W Bell, Model 7010) which is based on the use of an Hall probe to measure the intensity of the magnetic field. The distance between the coil and the probe is controlled by a 3-axis manual manipulator 
(Newport Corporation). The current applied to the coil is set to $0.4 \mathrm{~A}$ which is a typical value for the setup. Figure 4

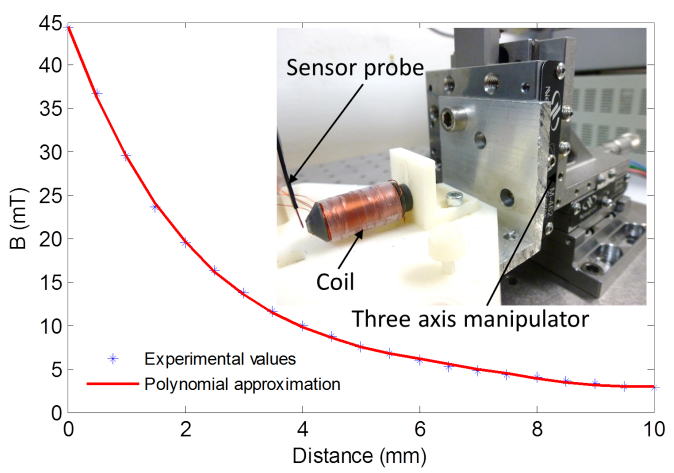

Fig. 4. Experimental setup used for the magnetic field measurement along the coil axis: an Hall sensor measures the magnetic field. A 3 axis manipulator controls the distance between the coil and the magnetic probe.

shows the experimental measurements. The minimum value of the magnetic field is measured at a distance of $10 \mathrm{~mm}$ from the coil and is about $2.9 \mathrm{mT}$. Therefore, the minimum external induction is about $2308 \mathrm{~A} / \mathrm{m}$ which is greater than the value of the coercive induction for a nickel sample [14], [15]. The magnetization of the nickel sphere is thus saturated in the whole workspace. To calculate the magnetic gradient field from the magnetic field measurements the experimental measurements are approximated by a fourth order polynomial, which is then differentiated.

\section{B. Simulation of the motion of the microrobot}

The motion of the microrobot is analysed using a numerical simulation software (Matlab) based on the dynamic model (5). The numerical values used are given in Table I. Figure 5 represents the frequency of motion $f$ simulated for a scale factor $a=10$ and an offset distance $d=4 \mathrm{~mm}$. This numerical solution is compared to the analytical solutions (15) and (18) developed in Section III also represented in Figure 5.

The trends of the analytical solution derived for small and large radius microrobots are similar to the numerical simulation. The main results of Section III are thus confirmed: (i) for small microrobots the inertial force is negligible, and (ii) for large microrobots the viscous force is negligible. Several reasons can explain the differences between the analytical and the numerical solutions. The first one is that the magnetic field gradient has been considered as constant to derive the analytical solution. The second one is that the inertia of the liquid is neglected in the simplified dynamic model. The model can be improved by considering a converted mass of the microrobot in (17).

The optimal radius obtained by the numerical simulation is equal to $22 \mu \mathrm{m}$ in water and corresponds to a maximum frequency $f_{\max }=127 \mathrm{~Hz}$. A ferromagnetic microrobot of $22 \mu \mathrm{m}$ size will be able to perform 127 cycles of back-andforth motion per second in its workspace, and thus to handle a large number of micro-objects individually in a short time, for applications such as assembly of microcomponents and cell transportation.

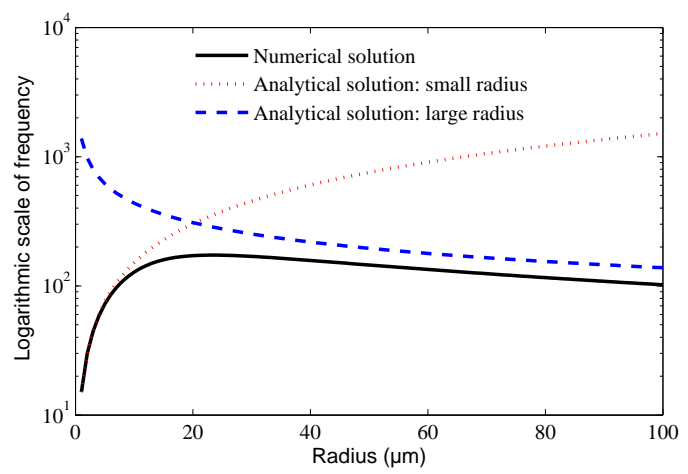

Fig. 5. Frequency of motion $f$ derived by the analytical and the numerical approaches. The maximal frequency derived by the analytical approach $f_{\max }=306 \mathrm{~Hz}$ is obtained for an optimal radius $r_{o p}=20 \mu \mathrm{m}$. The numerical simulation gives a maximal frequency $f_{\max }=127 \mathrm{~Hz}$ for an optimal radius $r_{o p}=22 \mu \mathrm{m}$. Trends of the curves and order of magnitude of the frequency and of the optimal radius are thus similar.

Figure 6 shows the analytical curves and the numerical values of the optimal radius calculated for different geometries of the workspace (parameters $a$ and $d$ ). Based on the analytical expressions (15), (18) and (20) the increase of the scale factor $a$ decreases the maximum frequency of motion but increases the value of the optimal radius. If the distance $d$ between the coil and the workspace increases the microrobot is subject to a lower magnetic field gradient $\left(\beta\right.$ and $\beta_{0}$ decreases when $d$ increases). According to (15), (18) and (20) the frequency $f$ will decrease while the optimal radius will increase. The numerical simulation confirms these trends. However, the difference between the analytical curves and the simulated values of the optimal radius is important for large scale factors. Indeed, the analytical results are obtained assuming small displacement around the initial position $(x=$ $d+a r)$, which holds only for small scale factors.

The use of different liquid environments changes the behavior of the microrobots due to the modification of the dynamic viscosity $\eta$. Figure 7 illustrates this result. For viscous environments the response time of the system increases (the acceleration decreases) which corresponds to slower motions. Thus, the average velocity $V_{m}$ and the motion frequency $f$ decrease. The simulated results show that the optimal radius for a blood environment is equal to three times the one for a water environment. This is in good agreement with the analytical expression (20) since a factor of 3.3 was predicted by the term $\eta^{\frac{2}{3}}$.

The numerical simulation has thus confirmed the trends of the analytical expressions obtained from a simplified model. These expressions can be used to derive rules to develop microrobots for high throughput handling of micro-objects.

\section{CONCLUSiON}

In this work a general approach has been proposed to optimize the size of a ferromagnetic microrobot aiming to 


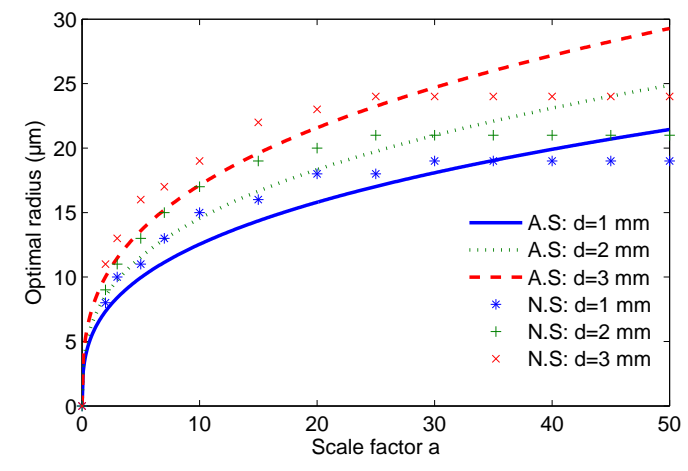

Fig. 6. Optimal radius $r_{o p}$ computed as a function of the workspace size $a$ for different offsets $d$ obtained by the analytical and the numerical approaches (A.S: analytical solution, N.S: numerical solution).

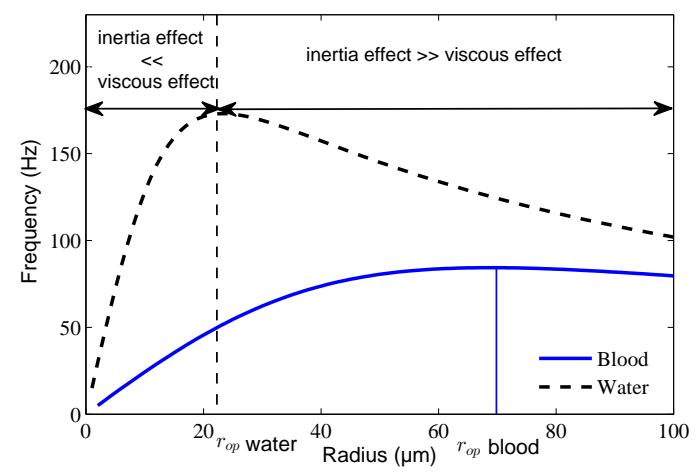

Fig. 7. Impact of the liquid medium viscosity on the frequency of motion $f$ For a water medium the maximum frequency is $f_{\max }=127 \mathrm{~Hz}$ for an optimal radius $r_{o p}=22 \mu \mathrm{m}$. For a blood medium $f_{\text {max }}=84 \mathrm{~Hz}$ and $r_{o p}=68 \mu \mathrm{m}$. The scale factor $a$ is 10 and the offset $d$ is $4 \mathrm{~mm}$.

maximize its dynamic capacity of displacement. The optimization method presented in this paper can be applied for different shapes of microrobot, but it has been illustrated for a spherical microrobot. The analytical approach demonstrates that there is a trade off between the viscous forces ruling the behavior of small microrobots and the inertial effects limiting the motion of large ones. An optimal radius of a few tens of micrometers approximated by the limit between the two behaviors has been derived. The frequency of motion obtained for the microrobots of optimal size is more than $100 \mathrm{~Hz}$, which is highly promising for applications that necessitate to handle a large number of micro-objects. Good agreement between the analytical approach and the simulation results (that do not assume neither a constant gradient magnetic field nor a Stokes flow) is highlighted, which validates the approximation made in the analytical analysis. The influence of the scale factor, the offset distance and the dynamic viscosity has been studied. This theoretical work shows a general method that can be applied to identify the best microrobot size when developing a wireless magnetic device. Therefore, the magnetic device can be used for any application requiring to handle a large number of objects, such as cells sorting for example.

This modeling and simulation work is a preliminary work, which will be validated experimentally in future works using microrobots with sizes ranging from a few micrometers to several hundred micrometers with different shapes.

\section{Acknowledgments}

This work has been supported by the French Agence Nationale de la Recherche, through the LEMA project (contract "ANR 12 BS03 007 01"), by the Labex ACTION project (contract "ANR-11-LABX-01-01") and by the "Région Franche-comté".

\section{REFERENCES}

[1] B. J. Nelson, I. K. Kaliakatsos, and J. J. Abbott, "Microrobots for minimally invasive medicine," Annual review of biomedical engineering, vol. 12 , pp. $55-85,2010$

[2] T. Kawahara, M. Sugita, M. Hagiwara, Y. Yamanishi, F. Arai, H. Kawano, I. Shihira-Ishikawa, and A. Miyawaki, "On-chip manipulation and sensing of microorganisms by magnetically driven microtools with a force sensing structure," in IEEE International Conference on Robotics and Automation, 2012, pp. 4112-4117.

[3] H. Uvet, L. Feng, S. Ohashi, M. Hagiwara, T. Kawahara, Y. Yamanishi, and F. Arai, "On-chip single particle loading and dispensing," in IEEE International Conference on Robotics and Automation, 2011, pp. 3151-3156.

[4] N. Inomata, T. Mizunuma, Y. Yamanishi, S. Kudo, and F. Arai, "Onchip magnetically driven micro-robot for enucleation of oocyte," in International Symposium on Micro-NanoMechatronics and Human Science, 2009, pp. 493-498.

[5] B. R. Donald, C. G. Levey, and I. Paprotny, "Planar microassembly by parallel actuation of mems microrobots," Microelectromechanical Systems, Journal of, vol. 17, no. 4, pp. 789-808, 2008.

[6] I. A. Ivan, G. Hwang, J. Agnus, M. Rakotondrabe, N. Chaillet, and S. Régnier, "First experiments on magpier: a planar wireless magnetic and piezoelectric microrobot," in IEEE International Conference on Robotics and Automation, 2011, pp. 102-108.

[7] C. Pawashe, S. Floyd, E. Diller, and M. Sitti, "Two-dimensional autonomous microparticle manipulation strategies for magnetic microrobots in fluidic environments," IEEE Transactions on Robotics, pp. 467-477, 2012.

[8] M. S. Sakar, E. B. Steager, A. Cowley, V. Kumar, and G. J. Pappas, "Wireless manipulation of single cells using magnetic microtransporters," in IEEE International Conference on Robotics and Automation, 2011, pp. 2668-2673.

[9] S. Bouchebout, A. Bolopion, J.-O. Abrahamians, and S. Régnier, "An overview of multiple dof magnetic actuated micro-robots," Journal of Micro-Nano Mechatronics, vol. 7, no. 4, pp. 97-113, 2012.

[10] D. H. Kim, P. S. S. Kim, A. A. Julius, and M. J. Kim, "Threedimensional control of engineered motile cellular microrobots," in IEEE International Conference on Robotics and Automation, 2012, pp. 721-726.

[11] K. B. Yesin, K. Vollmers, and B. J. Nelson, "Modeling and control of untethered biomicrorobots in a fluidic environment using electromagnetic fields," The International Journal of Robotics Research, vol. 25, no. 5-6, pp. 527-536, 2006.

[12] L. Zeng, S. Balachandar, and P. Fischer, "Wall-induced forces on a rigid sphere at finite reynolds number," Journal of Fluid Mechanics, vol. 536, no. 1, pp. 1-25, 2005.

[13] M. P. Kummer, J. J. Abbott, B. E. Kratochvil, R. Borer, A. Sengul, and B. J. Nelson, "Octomag: An electromagnetic system for 5-dof wireless micromanipulation," IEEE Transactions on Robotics, pp. 1006-1017, 2010.

[14] C. Graham and B. Lorenz, "Demagnetizing factors for disk samples of nonideal soft magnetic materials," Journal of Applied Physics, vol. 107, no. 9, pp. 09A322-09A322, 2010.

[15] K. Mulyokov, G. Korznikov, R. Abdulov, and R. Valiev, "Magnetic hysteretic properties of submicron grained nickel and their variations upon annealing," Journal of magnetism and magnetic materials, vol. 89, no. 1-2, pp. 207-213, 1990. 